\title{
The Impact of Microfinance on Poverty Alleviation: The Case of Pakistan
}

\author{
Wook Sohn (Corresponding author) \\ Professor, KDI School of Public Policy and Management \\ E-mail: wooksohn@kdischool.ac.kr \\ Laila Ume \\ Assistant Professor, National University of Sciences and Technology \\ E-mail: dr.umelaila@s3h.nust.edu.pk
}

Received: June 20, 2019 Accepted: July 21, 2019 Published: July 29, 2019

doi:10.5296/jad.v5i3.15165 URL: https://doi.org/10.5296/jad.v5i3.15165

\begin{abstract}
This paper examines the effect of microfinance on poverty alleviation in Pakistan using district-level panel data. We conduct fixed effect panel regressions to show a statistically significant positive impact of microfinance on household income, ownership of household assets, education, and expenditure. These results suggest that the development of microfinance is an efficient tool for both welfare improvement and poverty alleviation in Pakistan.
\end{abstract}

Keywords: Microfinance institutions, household income, poverty alleviation, economic development, Pakistan

JEL classification: $\mathrm{O} 12, \mathrm{O} 16$

\section{Introduction}

The main objective of microfinance is to increase the outreach of financial services to ensure poverty alleviation. Microfinance provides people in poverty with an opportunity to obtain small-scale monetary services, such as credit, savings, and insurance, because they lack access to such services from formal banking and other financial systems (Morduch, 1999a). Recently, there has been a shift from the social objective to the economic objective of sustainability of market-based financial services for the poor (Rhyne, 1998). 
Several studies have analyzed the impact of microfinance on poverty reduction. Soulama (2005) argues that microfinance is a unique development tool that works toward reducing poverty and maintaining self-sustainability. Microfinance not only supports the poor by providing them with capital but also helps increase their business opportunities, which improves personal income and increases personal investment in family health, nutrition, education, etc. (Colman, 2006; Morduch, 2000). Poor clients can apply for small loans and start their own businesses, such as small grocery stores, micro-enterprises, and fisheries; in this way, they are able to enhance their income sources and entrepreneurship skills. Microfinance is also helpful in empowering women (Pitt et al., 2006; Khandker and Pitt, 1998) and supporting poor people through group lending, which increases community development (Coleman, 1999; Barboza \& Trejos, 2009; Osmani, 2007).

Many studies have found positive and significant impacts of microfinance programs on the poor from various aspects. Microfinance can be a successful strategy for providing financial services (Miller \& Martinez, 2006; Stephens \& Tazi, 2006). The implementation of microfinance programs helps raise the income and living standard of the poor while boosting the local economy (Khandker, 2005). Khandker and Pitt (1998) argue that microfinance programs encourage investment in human capital by providing the poor with a choice in schooling. According to Hossain (1988), microfinance has a positive effect on certain social aspects, such as empowerment and self-confidence.

In Pakistan, poverty has increased almost constantly since the country's independence in 1947, except during 1980-1988, when poverty declined in urban and rural areas. Microfinance is becoming an important solution for alleviating poverty, through improving access to health services, children's education, food, and better household conditions for microfinance clients (Ahmad et al., 2004). It is worth noting that women are playing an important role in the growth and stability of microenterprises in Pakistan, which, in turn, contribute to the economic development of the country.

The primary objective of this paper is to evaluate the effect of microfinance programs on poverty using multidimensional poverty measures in Pakistan. In this study, we examine empirically the association between microfinance and living standards and evaluate the extent to which microfinance can be a tool for improving education, health, and social status.

Many studies have investigate the above research questions from experiences in various countries. First, food, clothing, and shelter are considered as primary necessities for humans, whereas employment, education, and utilities are regarded as secondary requisites for living (Doocy et al., 2005; Johnson \& Roglay, 1997). Microfinance is a source for improving living standards by providing all these essentials to the poor and alleviating hunger at individual and household levels (Amjad \& Kemal, 1997; Khandker, 2005).

Second, financial facilities, such as loans, deposits, and remittances, enable households to benefit from productive ventures, ease consumption throughout seasonal income flows, and help mitigate risks (Elbers et. al., 2003; Sharma \& Zeller, 1999). However, as stated by Conning and Udry (2007), poor households generally have limited access to formal financial markets due to the lack of information, incentives, and contract enforcement. 
Third, microfinance not only provides financial services but also empowers women. Microfinance clients usually have insufficient resources to start their own business, even though they are self-employed entrepreneurs; thus, they are unable to come out of poverty. In this situation, microfinance is a very effective development tool, providing the clients with small loans without providing collateral (Soulama, 2005; Brau \& Woller, 2004).

Fourth, education is an extremely important factor for the improvement of livelihoods and the advancement of human beings. Children of microfinance borrowers are better at learning and are more punctual (Ali \& Thorbecke, 2000). According to Psacharopoulos and Woodhall (1993), microfinance has a positive effect on poverty reduction and educational improvement. Microfinance programs also positively affect school enrollment and wellbeing (Morduch, 2009b; Wright, 2000).

Fifth, health is a major factor in assessing the welfare conditions of the poor and is one of the major dimensions of poverty and a primary non-economic outcome of microfinance. Poor people cannot fulfill their health requirements due to lack of resources (Collins et. al., 2009). Microfinance programs improve poor people's access to healthcare services and make health-related precautionary measures more affordable, although few studies have proven this connection. Gopalan (2007) shows that microfinance improved health conditions of poor borrowers and Darley-Harris (2002) reports that microfinance produces positive outcomes in terms of borrower's literacy regarding health issues.

We use fixed effect panel regression to examine the effect of microfinance on the household income and living standards of 94 districts in four provinces, including both urban and rural areas of Pakistan, from 2006 to 2014. This study employs multidisciplinary poverty measures to analyze Pakistan's microfinance sector.

There are reasons for choosing Pakistan for our study. First, although Pakistan is a fresh entrant in microfinance compared to India and Bangladesh, Pakistan is usually mentioned as a "regional leader" in establishing the latest versions of microfinance banks (MFBs). (Note 1) According to the Economist Intelligence Unit, in its annual review, Pakistan is among very few economies that have formulated a microfinance regulatory framework for MFBs. Similarly, the Consultative Group to Assist the Poor (CGAP), a research center at the World Bank, stated in 2011 that the microfinance sector in Pakistan is a "laboratory for innovation".

Second, due to the economic and political crises and external and internal security issues, Pakistan is currently experiencing sluggish development and increasing poverty. Thus, it is widely believed that microfinance is less susceptible to these political and economic barriers to development (Swain \& Floro, 2008). An extensive study on microfinance and conflicts claims that, by supporting reconstruction through the establishment of local microfinance, a country's economy can jump-start both during and after a conflict (Doyle, 1998; Nagarajan \& McNulty, 2004; Manalo, 2003).

The rest of this paper is organized as follows. Section 2 reviews the econometric models that are used to analyze the effect of microfinance on educational level, income, and living standards. The data are explained in Section 3. The results of the empirical analysis are 
presented in Section 4. The paper ends with a discussion and concluding remarks in Section 5 .

\section{Data}

In this study, we use the annual data from 2006 to 2014 for 94 districts in four provinces, including both urban and rural areas of Pakistan. Our district-level data are obtained from the Pakistan Microfinance Network (PMN), the Pakistan Social and Living Standards Measurement Survey (PSLM) of the Pakistan Bureau of Statistics, and the Human Development Index. Some policy variables are taken from Pakistan's Provincial Development Reports.

The Pakistan Microfinance Network (PMN) provides complete information on 12 microfinance providers. (Note 2) It provides data on microfinance scale, outreach, insurance, targets, financial performance, and sustainability of MFIs. The PSLM provides social and economic indicators that measure the welfare of society at provincial and district levels. The PSLM surveys are developed to assess poverty and other development issues at the district level, offering various indicators, such as income, health, education, living standards, household assets, welfare, sanitation, and physical environment. (Note 3)

The data in our study have some limitations. Our data are based on the district level, and some important variables that can measure the incidence and depth of poverty are not available at the district level. The PMN collects data from MFIs about microfinance outreach, and the quality of the data might be low compared to larger datasets. Thus, to remove errors from the dataset, we remove the outliers from our data. Furthermore, the PMN does not provide data on clients' poverty levels, which is a chief limitation of our study. PSLM data are reliable, but many variables that provide good measures for poverty are not available at the district level.

Our data are obtained from the PMN, which provides quarterly data, and from the PSLM, which provides bi-yearly data; both are at the district level. In other words, our data are mismatched by period. We run the following regression to generate the missing values:

$$
Y=\beta_{0}+\beta_{1} \text { district }+\beta_{2} \text { time }+\mu
$$

where $Y$ denotes the indicators of the social and economic status of a household. (Note 4). We use mean values for the imputation of missing values in the model, which may produce large significance levels and low standard errors, thus over-fitting the data.

Table 1 presents the evolution of microfinance activities in Pakistan from 2009 to 2014. The number of active borrowers doubled, from 1.4 million to 2.8 million people, and, in particular, the number of active women borrowers nearly tripled, from 0.6 million to 1.6 million, during the period. Gross loan portfolio, total assets, and deposits also increased significantly. There was also a significant rise in the number of branches and the number of total staff. 
Table 1. Microfinance in Pakistan

\begin{tabular}{|c|c|c|c|c|c|c|}
\hline & 2009 & 2010 & 2011 & 2012 & 2013 & 2014 \\
\hline No. of active borrowers (in millions) & 1.4 & 1.6 & 1.7 & 2.0 & 2.4 & 2.8 \\
\hline Gross loan portfolio (PKR billions) & 16.8 & 20.2 & 24.8 & 33.1 & 46.6 & 61.1 \\
\hline $\begin{array}{l}\text { No. of active women borrowers (in } \\
\text { millions) }\end{array}$ & 0.6 & 0.8 & 0.9 & 1.3 & 1.4 & 1.6 \\
\hline No. of branches & 1,221 & 1,405 & 1,550 & 1,460 & 1,606 & 1,747 \\
\hline No. of total staff & 11,557 & 12,005 & 14,202 & 14,648 & 17,456 & 19,881 \\
\hline Total assets (PKR billions) & 30.4 & 35.8 & 48.6 & 61.9 & 81.5 & 100.7 \\
\hline Deposits (PKR billions) & 7.2 & 10.1 & 13.9 & 20.8 & 32.9 & 42.7 \\
\hline Total debt (PKR billions) & 23.2 & 27.5 & 38.3 & 24.9 & 26.9 & 31.1 \\
\hline Total revenue (PKR billions) & 6.4 & 7.5 & 10.1 & 12.5 & 17.3 & 24.3 \\
\hline
\end{tabular}

Source: Pakistan Microfinance Review 2014

Table 2. Classification of Microfinance Providers

\begin{tabular}{|c|c|c|}
\hline Classification & Characteristics & Examples \\
\hline \multirow[t]{5}{*}{ Formal } & Full service, broad Spectrum & Bank of Khyber \\
\hline & Full service microfinance specialists & First Microfinance Bank \\
\hline & $\begin{array}{l}\text { Restricted service microfinance, broad } \\
\text { spectrum }\end{array}$ & National rural support program \\
\hline & $\begin{array}{l}\text { Restricted } \\
\text { specialist }\end{array}$ service microfinance & Kashf Foundation \\
\hline & Apex institutions & Pakistan poverty alleviation fund \\
\hline \multirow[t]{4}{*}{ Informal } & Community-based services & Rotating saving and credit association \\
\hline & & $\begin{array}{l}\text { Accumulating Savings and Credit } \\
\text { Associations(ASCAs) }\end{array}$ \\
\hline & Commercial services & Money lenders, stores \\
\hline & Family and friends & Mr. and Mrs. Hassan of Mingora \\
\hline
\end{tabular}

Source: Poverty and Social Impact Assessment

Table 2 shows that there are two primary classifications of microfinance: formal and informal. Each type has different level of services - full services with a broad spectrum, restricted services, and community-based services. Formal providers are professional financial service organizations, such as banks, whereas informal providers consist of friends and family, landlords, money lenders, and community-based services. This table also provides examples 
of formal and informal MFIs operating currently in Pakistan. The poor depend more upon informal microfinance providers to meet their credit requirements because informal providers are more easily accessible than are formal providers.

Table 3 presents the summary statistics of some variables used in the paper. It shows the mean, standard deviation, minimum, and maximum for variables of interest.

Table 3. Summary statistics of selected variables

\begin{tabular}{llllll}
\hline Variables & Obs. & Mean & Std. Dev. & Min & Max \\
\hline No. of active borrowers & 466 & 18433.44 & 27124.83 & 8 & 222800 \\
Gross loan portfolio & 468 & $2.34 \mathrm{e}+08$ & $3.51 \mathrm{e}+08$ & 652 & $2.52 \mathrm{e}+09$ \\
No. of active savers & 401 & 48686.44 & 308232.1 & 0 & 5982607 \\
Value of savings & 400 & $1.12 \mathrm{e}+08$ & $4.95 \mathrm{e}+08$ & 0 & $8.31 \mathrm{e}+09$ \\
Education & 311 & 103.0708 & 22.71491 & 20 & 128 \\
Health & 301 & 6.049231 & $2.90 \mathrm{e}+00$ & 0 & 18 \\
Wealth & 319 & 12.26154 & 13.10013 & 0 & 68 \\
\hline
\end{tabular}

Source: Pakistan Microfinance Connect and Pakistan Bureau of Statistics

\section{Econometric Models}

In this study, we identify various aspects by which microfinance affects poverty. We consider five dimensions to poverty: education, health, living standards, wealth, and consumption. We examine the effect of microfinance on poverty by using the multidimensional poverty measures. They include years of schooling, enrollment rate, and school attendance as indicators for education; health consultation as an indicator for health; house construction material and electricity as indicators for living standards; ownership of assets as an indicator for wealth; and food consumption and the number of meals taken per day as an indicator for consumption.

More specifically, we examine an association between the multidimensional poverty measures and microfinance activities, which are indicated by the number of active borrowers, the number of active savers, the value of savings, and the gross loan portfolio at the district level in Pakistan using the following pooled OLS:

$$
Y_{i t}=X^{\prime} \beta+P_{\alpha}+T_{\gamma}+\varepsilon_{i t}
$$

where $Y_{i t}$ represents the indicators of multidimensional poverty. First, we use education measures, including the number of people attending school, the number of people who have completed high school education, gross and net enrollment rates at primary schools, and gross and net enrollment rates at primary schools in slum areas Katchi Abadis. Second, we consider health and living standards, using health consultation, house roofing material (whether wood or bamboo), livestock ownership, and house ownership. Third, we use income 


\section{Al Macrothink}

and food consumption measures, such as economic status, meals taken per day, and consumption of food. $X$ denotes explanatory variables representing microfinance activities, including the number of active borrowers from MFIs, the number of active savers, the value of the savings of borrowers, and the gross loan portfolio. We also consider various controls when constructing regression equations. $P_{\alpha}$ is a provincial dummy variable and $T_{\gamma}$ is a time dummy. $\varepsilon_{i t}$ is an error term assumed to be white noise. We apply log-log for all estimations to analyze the long-term impact of the microfinance activities on multidimensional poverty. Table 4 describes the definition of variables and the data sources of variables used for our analysis.

Table 4. Definition of Variables

\begin{tabular}{|c|c|c|c|}
\hline Variables & Definition & Rationale & Data Source \\
\hline No. of active borrowers & $\begin{array}{l}\text { The numbers of individuals who } \\
\text { currently have an outstanding } \\
\text { loan balance with an MFI or are } \\
\text { responsible for repaying any } \\
\text { portion of the gross loan } \\
\text { portfolio. }\end{array}$ & $\begin{array}{l}\text { Captures the } \\
\text { micro-credit outreach }\end{array}$ & $\begin{array}{l}\text { PMN ; Micro Watch } \\
(2014)\end{array}$ \\
\hline No. of active savers & $\begin{array}{l}\text { The number of individuals who } \\
\text { currently have placed funds with } \\
\text { an MFI. This number applies } \\
\text { only to savings held by an MFI, } \\
\text { not to those savings held in other } \\
\text { institutions by the MFI's clients. }\end{array}$ & $\begin{array}{l}\text { Measures the } \\
\text { microcredit outreach }\end{array}$ & $\begin{array}{l}\text { PMN ; Micro Watch } \\
\text { (2014) }\end{array}$ \\
\hline Value of savings & $\begin{array}{l}\text { The total value of funds placed in } \\
\text { an account with an MFI that are } \\
\text { payable to a saver. }\end{array}$ & $\begin{array}{l}\text { Captures the } \\
\text { microcredit outreach }\end{array}$ & $\begin{array}{l}\text { PMN ; Micro Watch } \\
\text { (2014) }\end{array}$ \\
\hline Gross loan portfolio & $\begin{array}{l}\text { All outstanding principal for all } \\
\text { outstanding client loans, } \\
\text { including current, delinquent, } \\
\text { and restructured loans, but not } \\
\text { loans that have been written-off. } \\
\text { It does not include interest } \\
\text { receivable. It does not include } \\
\text { employee loans. }\end{array}$ & $\begin{array}{ll}\text { Measures the } & \text { the } \\
\text { microcredit activity } & \end{array}$ & $\begin{array}{l}\text { PMN ; Micro Watch } \\
\text { (2014) }\end{array}$ \\
\hline Monthly wage & $\begin{array}{l}\text { Percentage distribution of } \\
\text { monthly household income }\end{array}$ & $\begin{array}{l}\text { A good proxy for } \\
\text { poverty }\end{array}$ & PSLM (2014) \\
\hline $\begin{array}{l}\text { Adult literacy population } \\
15 \text { years and older }\end{array}$ & $\begin{array}{l}\text { Population aged } 15 \text { years and } \\
\text { older that is literate expressed as } \\
\text { percentage of the population } \\
\text { aged } 15 \text { years and older. }\end{array}$ & $\begin{array}{l}\text { Measures the educated } \\
\text { population }\end{array}$ & PSLM (2014) \\
\hline $\begin{array}{l}\text { Households by material } \\
\text { used for roofing by } \\
\text { RCC/RBC }\end{array}$ & $\begin{array}{l}\text { Percent distribution of } \\
\text { households by material used for } \\
\text { roofing by RCC/RBC }\end{array}$ & $\begin{array}{l}\text { Captures the household } \\
\text { condition }\end{array}$ & PSLM (2014) \\
\hline One room in household & $\begin{array}{l}\text { Percentage distribution of } \\
\text { households with one room. }\end{array}$ & $\begin{array}{l}\text { Measures the size of } \\
\text { house }\end{array}$ & PSLM (2014) \\
\hline
\end{tabular}




\begin{tabular}{|c|c|c|c|}
\hline Livestock ownership & 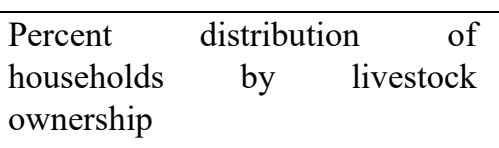 & $\begin{array}{l}\text { Measures the wealth } \\
\text { status }\end{array}$ & PSLM (2014) \\
\hline Ownership of house & $\begin{array}{l}\text { Percent distribution of } \\
\text { households by house ownership }\end{array}$ & Captures the wealth & PSLM (2014) \\
\hline $\begin{array}{l}\text { Ownership of household } \\
\text { assets }\end{array}$ & $\begin{array}{l}\text { Percent } \\
\text { households by household } \\
\text { ownership }\end{array}$ & $\begin{array}{l}\text { Captures the wealth } \\
\text { status }\end{array}$ & PSLM (2014) \\
\hline $\begin{array}{l}\text { Distribution } \\
\text { household material used } \\
\text { for roof by wood } \\
\text { /bamboo }\end{array}$ & $\begin{array}{l}\text { Percent distribution of } \\
\text { households by material used for } \\
\text { roof by wood/bamboo }\end{array}$ & $\begin{array}{l}\text { Captures the household } \\
\text { condition }\end{array}$ & PSLM (2014) \\
\hline $\begin{array}{l}\text { Households by other for } \\
\text { lighting }\end{array}$ & $\begin{array}{l}\text { Percent distribution } \\
\text { households by other } \\
\text { electricity for lighting }\end{array}$ & $\begin{array}{l}\text { Captures the household } \\
\text { condition }\end{array}$ & PSLM (2014) \\
\hline Economic status & $\begin{array}{l}\text { Percent distribution of } \\
\text { households by the perception of } \\
\text { the economic situation of the } \\
\text { household }\end{array}$ & $\begin{array}{l}\text { A good proxy for } \\
\text { Poverty }\end{array}$ & PSLM (2014) \\
\hline $\begin{array}{l}\text { Higher school education } \\
\text { completed }\end{array}$ & $\begin{array}{l}\text { Percentage distribution of } \\
\text { population that has completed } \\
\text { higher level education }\end{array}$ & $\begin{array}{l}\text { Captures the education } \\
\text { status }\end{array}$ & PSLM (2014) \\
\hline $\begin{array}{l}\text { Attendance at primary } \\
\text { school }\end{array}$ & $\begin{array}{l}\text { Population that has ever } \\
\text { attended primary school }\end{array}$ & $\begin{array}{ll}\begin{array}{l}\text { Measures } \\
\text { educational level }\end{array} & \text { the }\end{array}$ & PSLM (2014) \\
\hline $\begin{array}{l}\text { Net enrollment rate at } \\
\text { primary level (age 6-10) }\end{array}$ & $\begin{array}{l}\text { Net enrollment rate at the } \\
\text { primary level (age 6-10) }\end{array}$ & $\begin{array}{ll}\text { Measures } & \text { the } \\
\text { educational level } & \end{array}$ & PSLM (2014) \\
\hline $\begin{array}{l}\text { Gross enrollment rate at } \\
\text { primary level in Katchi } \\
\text { Abadis }\end{array}$ & $\begin{array}{l}\text { Number of children attending } \\
\text { primary level (classes 1-5) } \\
\text { divided by number of children } \\
\text { aged } 6-10 \text { years multiplied by } \\
100 \text { enrollment in Katchi Abadis }\end{array}$ & $\begin{array}{l}\text { Measures the } \\
\text { educational level in a } \\
\text { slum area }\end{array}$ & PSLM (2014) \\
\hline $\begin{array}{l}\text { Net enrollment rate at } \\
\text { primary level in Katchi } \\
\text { Abadis }\end{array}$ & $\begin{array}{l}\text { Net enrollment rate at the } \\
\text { primary level (age 6-10) } \\
\text { enrollment in Katchi Abadis. }\end{array}$ & $\begin{array}{l}\text { Measures the } \\
\text { educational level in a } \\
\text { slum area }\end{array}$ & PSLM (2014) \\
\hline $\begin{array}{l}\text { No. of students attended } \\
\text { school }\end{array}$ & $\begin{array}{l}\text { Population that has ever } \\
\text { attended school }\end{array}$ & $\begin{array}{ll}\begin{array}{l}\text { Captures } \\
\text { attendance }\end{array} & \text { school }\end{array}$ & PSLM (2014) \\
\hline $\begin{array}{l}\text { Gross enrollment rate of } \\
\text { primary school level }\end{array}$ & $\begin{array}{l}\text { Number of children attending } \\
\text { primary level (classes 1-5) } \\
\text { divided by number of children } \\
\text { aged } 6-10 \text { years multiplied by } \\
100\end{array}$ & $\begin{array}{l}\text { Captures } \\
\text { educational level }\end{array}$ & PSLM (2014) \\
\hline $\begin{array}{l}\text { Completion of high school } \\
\text { education }\end{array}$ & $\begin{array}{l}\text { Percentage distribution of } \\
\text { population that has completed } \\
\text { high school education }\end{array}$ & $\begin{array}{l}\text { Measures the level of } \\
\text { education }\end{array}$ & PSLM (2014) \\
\hline
\end{tabular}




\begin{tabular}{lllrr}
\hline Health consultation & $\begin{array}{l}\text { Percentage distribution of } \\
\text { population under 5 years that } \\
\text { have fallen sick or been injured }\end{array}$ & status & the & PSLM (2014) \\
\hline Consumption of Food & $\begin{array}{l}\text { Monthly per capita consumption } \\
\text { of expenditure on major food } \\
\text { items }\end{array}$ & $\begin{array}{l}\text { Measures } \\
\text { consumption }\end{array}$ & the & PSLM (2014) \\
\hline No. of meals taken per day & No. of meals taken per day & $\begin{array}{l}\text { Measures } \\
\text { consumption }\end{array}$ & food & PSLM (2014) \\
\hline
\end{tabular}

To overcome the estimation problem associated with omitted variables that can arise in pooled OLS estimation, we employ the fixed effect regression model to control for the unobservable variables that are constant over time but are different across districts. The regression equation can be shown as follows:

$$
Y_{i t}=X^{\prime} \beta+\mu_{i t}
$$

where $Y_{i t}$ represents the dependent variables for district $i$ for time $t, X$ is the vector of the explanatory variables, and $\mu_{i t}$ represents the stochastic error term.

$$
Y_{i t}=X^{\prime} \beta+v_{i}+\gamma_{t}+\varepsilon_{i t}
$$

We decompose the error term into three components, $\mu_{i t}=v_{i}+\gamma_{t}+\varepsilon_{i t}$, where $v_{i}$ represents the unobserved components that change across districts but are constant over time, $\gamma_{t}$ represents the unobserved factors that vary over time but are constant across districts, and $\varepsilon_{i t}$ represents all unobserved aspects or factors that change across time and the units of our study. We address potential heteroscedasticity by using clustered standard errors.

\section{Empirical Results}

We first analyze the long-term effect of microfinance on poverty alleviation in Pakistan using pooled OLS regressions. Table 5 and Table 6 illustrate the impact of microfinance on education and other variables, such as health, living standards, income, and food consumption.

In Table 5, the coefficients of the number of active borrowers are 0.014-0.314 and significant at either $5 \%$ or $10 \%$ levels in all specifications, suggesting that microfinance activities enhance education, as measured by the number of students attending school, the number of people with high school education completion, the enrollment rate in primary schools, and so on. Other measures of microfinance activities, such as gross loan portfolio and value of savings, show similar results. In Table 6 , the coefficients of the number of active borrowers, gross loan portfolio, and value of savings are all positive and significant at $1 \%$ or $5 \%$ levels in all specifications, suggesting that microfinance activities also improve health, living standards, income, and food consumption.

However, these results may have a problem associated with omitted variables arising from 
pooled OLS estimation. Therefore, we use a fixed effect regression model to control for the unobservable variables.

Table 5. Cross sectional regressions (education)

\begin{tabular}{|c|c|c|c|c|c|c|}
\hline & \multicolumn{6}{|l|}{ Education } \\
\hline & $\begin{array}{l}\text { No. of } \\
\text { students } \\
\text { attending } \\
\text { school }\end{array}$ & $\begin{array}{l}\text { Higher } \\
\text { school } \\
\text { education } \\
\text { completed }\end{array}$ & $\begin{array}{l}\text { Gross } \\
\text { enrollment } \\
\text { rate of } \\
\text { primary } \\
\text { school }\end{array}$ & $\begin{array}{l}\text { Net } \\
\text { enrollment } \\
\text { rate of } \\
\text { primary } \\
\text { school }\end{array}$ & $\begin{array}{l}\text { Gross } \\
\text { enrollment } \\
\text { rate } \\
\text { primary of } \\
\text { school in } \\
\text { Katchi } \\
\text { Abadis }\end{array}$ & $\begin{array}{l}\text { Net } \\
\text { enrollment } \\
\text { rate of } \\
\text { primary } \\
\text { school in } \\
\text { Katchi } \\
\text { Abadis }\end{array}$ \\
\hline & (1) & (2) & (3) & (4) & (5) & (6) \\
\hline $\begin{array}{ll}\text { Gross } & \text { loan } \\
\text { portfolio } & \end{array}$ & $\begin{array}{l}0.0192 * * \\
(0.0089)\end{array}$ & $\begin{array}{l}0.0121 * * \\
(0.0052)\end{array}$ & $\begin{array}{l}0.0272 * * \\
(0.0122)\end{array}$ & $\begin{array}{l}0.0136 * * \\
(0.0059)\end{array}$ & $\begin{array}{l}0.0142 * * \\
(0.0064)\end{array}$ & $\begin{array}{l}0.018 * * * \\
(0.0030)\end{array}$ \\
\hline $\begin{array}{l}\text { No. of active } \\
\text { borrowers }\end{array}$ & $\begin{array}{l}0.0256^{* *} \\
(0.0119)\end{array}$ & $\begin{array}{l}0.0191 * * \\
(0.0087)\end{array}$ & $\begin{array}{l}0.0147 * * \\
(0.0069)\end{array}$ & $\begin{array}{l}0.0159 * \\
(0.0085)\end{array}$ & $\begin{array}{l}0.0214 * * \\
(0.0101)\end{array}$ & $\begin{array}{l}0.0314 * \\
(0.0161)\end{array}$ \\
\hline $\begin{array}{l}\text { Value } \quad \text { of } \\
\text { savings }\end{array}$ & $\begin{array}{l}0.0177 * * \\
(0.0081)\end{array}$ & $\begin{array}{l}0.0256 * * \\
(0.0121)\end{array}$ & $\begin{array}{l}0.0296 * * \\
(0.0141)\end{array}$ & $\begin{array}{l}0.0131 * * \\
(0.0059)\end{array}$ & $\begin{array}{l}0.0256^{*} \\
(0.0132)\end{array}$ & $\begin{array}{l}0.0125 * * \\
(0.0061)\end{array}$ \\
\hline $\begin{array}{l}\text { One room in } \\
\text { household }\end{array}$ & $\begin{array}{l}-0.0156 * * * \\
(0.0001)\end{array}$ & $\begin{array}{l}-0.0315 * * * \\
(0.0002)\end{array}$ & $\begin{array}{l}-0.0278^{*} \\
(0.0131)\end{array}$ & $\begin{array}{l}-0.0368 * * \\
(0.0179)\end{array}$ & $\begin{array}{l}-0.0159 * * \\
(0.0071)\end{array}$ & $\begin{array}{l}-0.0102 * * \\
(0.0048)\end{array}$ \\
\hline $\begin{array}{l}\text { Livestock } \\
\text { ownership }\end{array}$ & $\begin{array}{l}0.5123 * * \\
(0.2492)\end{array}$ & $\begin{array}{l}0.486^{* *} \\
(0.2130)\end{array}$ & $\begin{array}{l}0.493 * * \\
(0.2310)\end{array}$ & $\begin{array}{l}0.265 * * \\
(0.1291)\end{array}$ & $\begin{array}{l}0.198 * * \\
(0.0910)\end{array}$ & $\begin{array}{l}0.315 * * \\
(0.1498)\end{array}$ \\
\hline $\begin{array}{l}\text { Adult literacy } \\
\text { population } 15 \\
\text { years and older }\end{array}$ & $\begin{array}{l}0.323 * * \\
(0.1521)\end{array}$ & $\begin{array}{l}0.351 * * * \\
(0.0004)\end{array}$ & $\begin{array}{l}0.440 * * \\
(0.2002)\end{array}$ & $\begin{array}{l}0.178 * * \\
(0.0810)\end{array}$ & $\begin{array}{l}0.342 * * \\
(0.1693)\end{array}$ & $\begin{array}{l}0.436 * * \\
(0.1972)\end{array}$ \\
\hline $\begin{array}{l}\text { Ownership of } \\
\text { house }\end{array}$ & $\begin{array}{l}0.0256 * * * \\
(0.0007)\end{array}$ & $\begin{array}{l}0.0412 * * * \\
(0.0018)\end{array}$ & $\begin{array}{l}0.0514 * * * \\
(0.0001)\end{array}$ & $\begin{array}{l}0.0314 * * * \\
(0.0017)\end{array}$ & $\begin{array}{l}0.0146 * * * \\
(0.0004)\end{array}$ & $\begin{array}{l}0.0241 * * * \\
(0.0007)\end{array}$ \\
\hline Monthly wage & $\begin{array}{l}0.4171 * * * \\
(0.0007)\end{array}$ & $\begin{array}{l}0.3795 * * * \\
(0.0021)\end{array}$ & $\begin{array}{l}0.2167 * * * \\
(0.0031)\end{array}$ & $\begin{array}{l}0.1163 * * * \\
(0.0107)\end{array}$ & $\begin{array}{l}0.2516 * * * \\
(0.0015)\end{array}$ & $\begin{array}{l}0.3165^{* *} \\
(0.1399)\end{array}$ \\
\hline $\begin{array}{l}\text { Households by } \\
\text { other } \\
\text { lighting }\end{array}$ & $\begin{array}{l}0.0871 * * * \\
(0.0003)\end{array}$ & $\begin{array}{l}0.0741 * * \\
(0.0299)\end{array}$ & $\begin{array}{l}0.0612 * * * \\
(0.0028)\end{array}$ & $\begin{array}{l}0.0359 * * \\
(0.0161)\end{array}$ & $\begin{array}{l}0.0161 * * * \\
(0.0072)\end{array}$ & $\begin{array}{l}0.0214 * * \\
(0.0092)\end{array}$ \\
\hline Constant & $\begin{array}{l}2.112 * * * \\
(0.0147)\end{array}$ & $\begin{array}{l}2.116^{* *} \\
(0.8206)\end{array}$ & $\begin{array}{l}3.165 * * \\
(1.1012)\end{array}$ & $\begin{array}{l}2.998 * * \\
(1.1059)\end{array}$ & $\begin{array}{l}4.065 * * \\
(1.9175)\end{array}$ & $\begin{array}{l}3.698 * * \\
(1.639)\end{array}$ \\
\hline $\begin{array}{l}\text { No. of } \\
\text { observations }\end{array}$ & 86 & 84 & 81 & 86 & 88 & 83 \\
\hline Adj. $\mathrm{R}^{2}$ & 0.411 & 0.331 & 0.421 & 0.355 & 0.246 & 0.316 \\
\hline
\end{tabular}

Notes. Robust clustered standard errors are in parenthesis. All variables are in natural logarithm. A time dummy and a district dummy are included in the estimation. *** $\mathrm{p}<0.01 ; * * \mathrm{p}<0.05 ; * \mathrm{p}<0.10$. 
Table 6. Cross sectional regressions (health and living standard; income and food consumption)

\begin{tabular}{|c|c|c|c|c|c|c|c|}
\hline & \multicolumn{4}{|c|}{ Health and living standard } & \multicolumn{3}{|c|}{ Income and food consumption } \\
\hline & $\begin{array}{c}\text { Health } \\
\text { consultation }\end{array}$ & $\begin{array}{l}\text { Household } \\
\text { material used } \\
\text { for roof by } \\
\text { wood/bamboo }\end{array}$ & $\begin{array}{l}\text { Livestock } \\
\text { ownership }\end{array}$ & $\begin{array}{l}\text { Ownership } \\
\text { of house }\end{array}$ & $\begin{array}{c}\text { Economic } \\
\text { status }\end{array}$ & $\begin{array}{l}\text { No. of } \\
\text { meals } \\
\text { taking per } \\
\text { day }\end{array}$ & $\begin{array}{c}\text { Consumption } \\
\text { of food }\end{array}$ \\
\hline & (1) & (2) & (3) & (4) & (5) & (6) & (7) \\
\hline $\begin{array}{l}\text { Gross loan } \\
\text { portfolio }\end{array}$ & $\begin{array}{c}0.0121 * * * \\
(0.0005)\end{array}$ & $\begin{array}{l}0.0133 * * \\
(0.0057)\end{array}$ & $\begin{array}{l}0.0141 * * \\
(0.0063)\end{array}$ & $\begin{array}{l}0.014 * * \\
(0.0062)\end{array}$ & $\begin{array}{l}0.023 * * \\
(0.0099)\end{array}$ & $\begin{array}{l}0.016 * * \\
(0.0068)\end{array}$ & $\begin{array}{l}0.0325 * * \\
(0.0157)\end{array}$ \\
\hline $\begin{array}{l}\text { No. of active } \\
\text { borrowers }\end{array}$ & $\begin{array}{l}0.0114 * * \\
(0.0049)\end{array}$ & $\begin{array}{l}0.0353 * * \\
(0.01657)\end{array}$ & $\begin{array}{l}0.0123 * * \\
(0.0052)\end{array}$ & $\begin{array}{l}0.0245^{* *} \\
(0.0101)\end{array}$ & $\begin{array}{l}0.0142 * * \\
(0.0052)\end{array}$ & $\begin{array}{l}0.0315^{* *} \\
(0.0091)\end{array}$ & $\begin{array}{c}0.0136 * * * \\
(0.0004)\end{array}$ \\
\hline $\begin{array}{l}\text { Value of } \\
\text { savings }\end{array}$ & $\begin{array}{l}0.0101 * * \\
(0.0042)\end{array}$ & $\begin{array}{c}0.0133 * * \\
(0.0054)\end{array}$ & $\begin{array}{l}0.0100 * * \\
(0.0030)\end{array}$ & $\begin{array}{c}0.0163 * * * \\
(0.0006)\end{array}$ & $\begin{array}{l}0.0201 * * \\
(0.0091)\end{array}$ & $\begin{array}{c}0.0123 * * \\
(0.0052)\end{array}$ & $\begin{array}{c}0.0221^{* * *} \\
(0.0006)\end{array}$ \\
\hline $\begin{array}{c}\text { One room in } \\
\text { household }\end{array}$ & $\begin{array}{c}-0.0125 * * * \\
(0.0002)\end{array}$ & $\begin{array}{c}-0.0225^{* * *} \\
(0.0015)\end{array}$ & $\begin{array}{c}-0.0315 * * * \\
(0.0012)\end{array}$ & $\begin{array}{c}-0.0258 * * \\
(0.0091)\end{array}$ & $\begin{array}{c}-0.0412 * * * \\
(0.0001)\end{array}$ & $\begin{array}{c}-0.0321 * * * \\
(0.0003)\end{array}$ & $\begin{array}{l}-0.0365^{* *} \\
(0.01423)\end{array}$ \\
\hline $\begin{array}{l}\text { Livestock } \\
\text { ownership }\end{array}$ & $\begin{array}{l}0.5327 * * \\
(0.2436)\end{array}$ & $\begin{array}{l}0.4195 * * \\
(0.1952)\end{array}$ & $\begin{array}{l}0.3961 * * \\
(0.1823)\end{array}$ & $\begin{array}{l}0.4720 * * \\
(0.1922)\end{array}$ & $\begin{array}{c}0.2953 * * \\
(0.1021)\end{array}$ & $\begin{array}{l}0.6321 * * \\
(0.2921)\end{array}$ & $\begin{array}{l}0.4023 * * \\
(0.1825)\end{array}$ \\
\hline $\begin{array}{c}\text { Adult literacy } \\
\text { population } 15 \\
\text { years and } \\
\text { older }\end{array}$ & $\begin{array}{l}0.3127 * * \\
(0.1398)\end{array}$ & $\begin{array}{l}0.1776 * * \\
(0.07139)\end{array}$ & $\begin{array}{l}0.1581 * * \\
(0.06122)\end{array}$ & $\begin{array}{l}0.2420 * * \\
(0.0980)\end{array}$ & $\begin{array}{l}0.1943 * * \\
(0.0811)\end{array}$ & $\begin{array}{l}0.2611 * * \\
(0.1219)\end{array}$ & $\begin{array}{l}0.1856^{* *} \\
(0.0813)\end{array}$ \\
\hline $\begin{array}{c}\text { Ownership of } \\
\text { house }\end{array}$ & $\begin{array}{l}0.0154 * * \\
(0.0061)\end{array}$ & $\begin{array}{l}0.0213 * * \\
(0.0891)\end{array}$ & $\begin{array}{l}0.0134 * * \\
(0.0051)\end{array}$ & $\begin{array}{l}0.0314 * * \\
(0.0131)\end{array}$ & $\begin{array}{l}0.0215^{* *} \\
(0.0083)\end{array}$ & $\begin{array}{l}0.0156^{* *} \\
(0.0053)\end{array}$ & $\begin{array}{c}0.0221 * * * \\
(0.0100)\end{array}$ \\
\hline $\begin{array}{l}\text { Monthly } \\
\text { wage }\end{array}$ & $\begin{array}{c}0.4136^{* * *} \\
(0.0001)\end{array}$ & $\begin{array}{c}0.3321^{* * * *} \\
(0.0002)\end{array}$ & $\begin{array}{c}0.4224 * * * \\
(0.0011)\end{array}$ & $\begin{array}{l}0.4196 * * \\
(0.1932)\end{array}$ & $\begin{array}{c}0.3321 * * * \\
(0.0001)\end{array}$ & $\begin{array}{c}0.3169 * * * \\
(0.0011)\end{array}$ & $\begin{array}{l}0.4013 * * \\
(0.1958)\end{array}$ \\
\hline $\begin{array}{l}\text { Households } \\
\text { by other for } \\
\text { lighting }\end{array}$ & $\begin{array}{l}0.0014 * * \\
(0.0005)\end{array}$ & $\begin{array}{l}0.0042 * * \\
(0.0016)\end{array}$ & $\begin{array}{l}0.0051 * * \\
(0.0017)\end{array}$ & $\begin{array}{l}0.0011 * * \\
(0.0002)\end{array}$ & $\begin{array}{l}0.0116 * * \\
(0.0043)\end{array}$ & $\begin{array}{l}0.0214 * * \\
(0.0101)\end{array}$ & $\begin{array}{l}0.0314 * * \\
(0.0131)\end{array}$ \\
\hline Constant & $\begin{array}{c}1.256^{*} \\
(0.6580)\end{array}$ & $\begin{array}{l}4.215^{* *} \\
(2.007)\end{array}$ & $\begin{array}{c}3.695^{*} \\
(1.9925)\end{array}$ & $\begin{array}{c}5.364 * \\
(2.7883)\end{array}$ & $\begin{array}{l}2.787 * * \\
(1.2013)\end{array}$ & $\begin{array}{c}6.325^{*} \\
(3.5321)\end{array}$ & $\begin{array}{c}2.325^{*} \\
(1.1988)\end{array}$ \\
\hline $\begin{array}{c}\text { No. of } \\
\text { observations }\end{array}$ & 77 & 81 & 73 & 89 & 81 & 81 & 81 \\
\hline Adj. $\mathrm{R}^{2}$ & 0.451 & 0.464 & 0.343 & 0.445 & 0.454 & 0.531 & 0.455 \\
\hline
\end{tabular}

Notes. Robust clustered standard errors are in parenthesis. All variables are in natural logarithm. Note: Robust clustered standard errors are in parenthesis. All variables are in natural logarithm. A time dummy and a district dummy are included in the estimation. *** $\mathrm{p}<0.01 ; * * \mathrm{p}<0.05 ; * \mathrm{p}<0.10$.

Table 7 demonstrates the impact of microfinance on various measure of education. Columns (1) and (2) represent the number of students attending school; columns (3) and (4) represent the number of people who have completed high school education; columns (5) and (6) represent the enrollment rate in primary schools. The adult literacy population 15 years and older (i.e., the population aged 15 years and older that is literate), the distribution of 
households with electric lighting, and the distribution of households based on the materials used for their roofing by RCC/RBC are used as control variables. The results show that a $10 \%$ increase in microfinance activities, measured by the number of active borrowers, the gross loan portfolio, and the value of savings, increases the number of students attending school and primary school enrollment by $0.11-0.22 \%$ and $0.13-0.21 \%$, respectively. The gross loan portfolio has a larger impact on levels of education than do either the number of active borrowers or the value of savings. In all specifications, the coefficients of microfinance activities are positive and statistically significant at $1 \%$ and $5 \%$ levels. These results suggest that microfinance helps people invest in human capital development and improve their education level, which plays a very important role in poverty alleviation in Pakistan.

Table 7. Fixed effect panel regressions (education)

\begin{tabular}{|c|c|c|c|c|c|c|}
\hline & \multicolumn{6}{|l|}{ Education } \\
\hline & \multicolumn{2}{|c|}{$\begin{array}{l}\text { No. of students } \\
\text { attending school }\end{array}$} & \multicolumn{2}{|c|}{$\begin{array}{l}\text { Higher school } \\
\text { education completed }\end{array}$} & \multicolumn{2}{|c|}{$\begin{array}{l}\text { Gross enrollment rate of } \\
\text { primary school }\end{array}$} \\
\hline & (1) & (2) & (3) & (4) & $(5)$ & (6) \\
\hline \multirow[t]{2}{*}{ Gross loan portfolio } & $0.022^{* * *}$ & & $0.033^{* *}$ & & $0.021^{* *}$ & \\
\hline & $(0.009)$ & & $(0.012)$ & & $(0.009)$ & \\
\hline \multirow[t]{2}{*}{ No. of active borrowers } & $0.012 * *$ & & $0.021^{* *}$ & & $0.013^{* *}$ & \\
\hline & $(0.006)$ & & $(0.008)$ & & $(0.008)$ & \\
\hline \multirow[t]{2}{*}{ Value of savings } & & $0.011 * *$ & & $0.014^{* *}$ & & $0.0155^{* *}$ \\
\hline & & $(0.005)$ & & $(0.005)$ & & $(0.006)$ \\
\hline \multirow{2}{*}{$\begin{array}{l}\text { Adult literacy population } 15 \\
\text { years and older }\end{array}$} & $0.684 * *$ & $0.649^{* *}$ & $0.794 * *$ & $0.812^{* *}$ & $0.394 * *$ & $0.360^{* *}$ \\
\hline & $(0.038)$ & $(0.045)$ & $(0.053)$ & $(0.051)$ & $(0.055)$ & $(0.061)$ \\
\hline \multirow[t]{2}{*}{ Households by other for lighting } & $0.035^{* *}$ & $0.040 * *$ & $0.028^{* *}$ & $0.033^{* *}$ & $0.063 * *$ & $0.060 * *$ \\
\hline & $(0.010)$ & $(0.012)$ & $(0.014)$ & $(0.014)$ & $(0.015)$ & $(0.016)$ \\
\hline \multirow{2}{*}{$\begin{array}{l}\text { Households by material used for } \\
\text { roof by } \mathrm{RCC} / \mathrm{RBC}\end{array}$} & $0.022 * *$ & $0.022 * *$ & $0.028^{* *}$ & $0.025^{* *}$ & $0.021^{* *}$ & $0.022^{* *}$ \\
\hline & $(0.008)$ & $(0.009)$ & $(0.010)$ & $(0.010)$ & $(0.011)$ & $(0.010)$ \\
\hline Provincial dummy & Yes & Yes & Yes & Yes & Yes & Yes \\
\hline Time dummy & Yes & Yes & Yes & Yes & Yes & Yes \\
\hline \multirow[t]{2}{*}{ Constant } & $1.144 * *$ & $1.194 * *$ & $0.395 * * *$ & $0.283 * *$ & $2.716^{* *}$ & $2.716^{* *}$ \\
\hline & $(0.135)$ & $(0.157)$ & $(0.189)$ & $(0.179)$ & $(0.197)$ & $(0.220)$ \\
\hline No. of observations & 254 & 238 & 254 & 238 & 254 & 238 \\
\hline Adj. $R^{2}$ & 0.772 & 0.753 & 0.772 & 0.753 & 0.772 & 0.753 \\
\hline
\end{tabular}

Notes. Robust clustered standard errors are in parenthesis. All variables are in natural logarithm. Coefficients are obtained using the fixed effect panel approach. A time dummy and a district dummy are included in the estimation. ${ }^{* * *} \mathrm{p}<0.01 ; * * \mathrm{p}<0.05 ; * \mathrm{p}<0.10$. 
Table 8 indicates the impact of microfinance on education from different perspectives. Columns (1) and (2) represent the net enrollment rate in primary schools; columns (3) and (4), represent the enrollment rate in primary schools in Katchi Abadis; and columns (5) and (6) represent the net enrollment rate in primary schools in the slum area. The results show that a $10 \%$ increase in microfinance activities, as measured by the number of active borrowers, the gross loan portfolio, and the value of savings, increases the net enrollment rate in primary schools by $0.14-0.19 \%$ and is statistically significant at the $5 \%$ level. It is particularly interesting to see in specifications (5)-(6) that the coefficients of those three microfinance measures in regressions of the net enrollment rate in primary schools in Katchi Abadis are 0.019-0.039, which is larger than are those in specifications (1)-(2). This result suggests that the impact of microfinance on education is enhanced in the slum area.

Table 8. Fixed effect panel regressions (education) -continued

\begin{tabular}{|c|c|c|c|c|c|c|}
\hline & \multicolumn{6}{|c|}{ Education } \\
\hline & \multicolumn{2}{|c|}{$\begin{array}{l}\text { Net enrollment rate } \\
\text { of primary school }\end{array}$} & \multicolumn{2}{|c|}{$\begin{array}{l}\text { Gross enrollment rate } \\
\text { of primary school in } \\
\text { Katchi Abadis }\end{array}$} & \multicolumn{2}{|c|}{$\begin{array}{l}\text { Net enrollment rate } \\
\text { of primary schoo } \\
\text { in Katchi Abadis }\end{array}$} \\
\hline & (1) & (2) & (3) & (4) & (5) & (6) \\
\hline \multirow[t]{2}{*}{ Gross loan portfolio } & $0.019 * *$ & & $0.026 * *$ & & $0.039 * *$ & \\
\hline & $(0.009)$ & & $(0.011)$ & & $(0.016)$ & \\
\hline \multirow[t]{2}{*}{ No. of active borrowers } & $0.014 * *$ & & $0.022 * *$ & & $0.036^{* *}$ & \\
\hline & $(0.006)$ & & $(0.008)$ & & $(0.008)$ & \\
\hline \multirow[t]{2}{*}{ Value of savings } & & $0.016^{* *}$ & & $0.018 * *$ & & $0.019 * *$ \\
\hline & & $(0.005)$ & & $(0.006)$ & & $(0.007)$ \\
\hline \multirow{2}{*}{$\begin{array}{l}\text { Adult literacy population } 15 \text { years and } \\
\text { older }\end{array}$} & $0.401 * *$ & $0.355^{* *}$ & $0.440 * *$ & $0.417 * *$ & $0.452 * *$ & $0.449 * *$ \\
\hline & $(0.051)$ & $(0.057)$ & $(0.058)$ & $(0.069)$ & $(0.059)$ & $(0.071)$ \\
\hline \multirow[t]{2}{*}{ Households by other for lighting } & $0.070 * *$ & $0.062 * *$ & $0.087 * *$ & $0.082 * *$ & $0.081 * *$ & $0.084 * *$ \\
\hline & $(0.014)$ & $(0.015)$ & $(0.016)$ & $(0.018)$ & $(0.016)$ & $(0.019)$ \\
\hline \multirow{3}{*}{$\begin{array}{l}\text { Households by material used for roof by } \\
\text { RCC/RBC }\end{array}$} & $0.019 * *$ & & & & & \\
\hline & $*$ & 0.013 & $0.028 * *$ & $0.026 * *$ & $0.034 * *$ & $0.028 * *$ \\
\hline & $(0.010)$ & $(0.009)$ & $(0.011)$ & $(0.011)$ & $(0.011)$ & $(0.012)$ \\
\hline Provincial dummy & Yes & Yes & Yes & Yes & Yes & Yes \\
\hline Time dummy & Yes & Yes & Yes & Yes & Yes & Yes \\
\hline \multirow[t]{2}{*}{ Constant } & $2.680 * *$ & $2.721 * *$ & $2.372 * *$ & $2.360 * *$ & $1.781 * *$ & $1.827 * *$ \\
\hline & $(0.179)$ & $(0.206)$ & $(0.207)$ & $(0.247)$ & $(0.211)$ & $(0.254)$ \\
\hline No. of observations & 254 & 238 & 254 & 238 & 254 & 238 \\
\hline Adj. $R^{2}$ & 0.612 & 0.604 & 0.612 & 0.604 & 0.612 & 0.604 \\
\hline
\end{tabular}

Notes. Robust clustered standard errors are in parenthesis. All variables are in natural logarithm. Coefficients are obtained using the fixed effect panel approach. A time dummy and a district dummy are included in the estimation. $* * * \mathrm{p}<0.01 ; * * \mathrm{p}<0.05 ; * \mathrm{p}<0.10$. 


\section{Macrothink

The adult literacy rate is used as a proxy for parents' education; Tables 7-8 show that the impact of parents' education on different levels of education is much higher than are those of any other explanatory variables. This result implies that educated parents tend to be more willing to invest in their children's education. Household conditions are used as a proxy to control for household living standards. These conditions include the presence of electricity for lighting and the material used for roofing and construction of houses. The impact of household conditions are also positive and statistically significant.

Table 9 illustrates the impact of microfinance on various measures of poverty alleviation: health, living standards, economic status, and food consumption. In columns (1) and (2), health condition is proxied by the proportion of households that consult a physician. (Note 5) The estimated results show that a $10 \%$ increase in microfinance activities, as measured by gross loan portfolio, the number of active borrowers, and the value of savings, increase health consultation by $0.11-0.14 \%$. In column 3 , the estimated coefficient of the value of savings is negative and significant at the 5\% level, showing that a higher value of savings can reduce the proportion of households who use either wood or bamboo for roofing materials. The results suggest that microfinance can be used as a vehicle for improving household living conditions. Columns (4)-(7) show the impact of microfinance on economic status and the number of meals taken per day. (Note 6) The estimated coefficients are positive and statistically significant at the $1 \%$ and $5 \%$ levels. We find that gross loan portfolio has a larger impact on economic status than do other microfinance measures, whereas the number of active borrowers has a larger impact on the number of meals taken per day than do other measures. These results suggest that the utilization of microfinance improves health, living standards, income, and food consumption.

Various measures of household living standards are used as control variables. They include the use of electricity for lighting, home ownership, and ownership of house assets, such as furniture, means of transportation, and appliances. The results show that the coefficients of home ownership and house assets are positive and statistically significant. 
Table 9. Fixed effect panel regressions (health and living standard; income and food consumption)

\begin{tabular}{|c|c|c|c|c|c|c|c|}
\hline & \multicolumn{2}{|c|}{ Health consultation } & \multirow{2}{*}{$\begin{array}{l}\text { Household } \\
\text { material used } \\
\text { for roof by } \\
\text { wood/bambo } \\
\text { o } \\
\text { (3) }\end{array}$} & \multicolumn{2}{|c|}{ Economic status } & \multicolumn{2}{|c|}{$\begin{array}{l}\text { No. of meals } \\
\text { taking per day }\end{array}$} \\
\hline & (1) & (2) & & (4) & $(5)$ & (6) & (7) \\
\hline \multirow[t]{2}{*}{ Gross loan portfolio } & $0.012 * *$ & & -0.022 & $\begin{array}{l}0.017 * \\
*\end{array}$ & & $\begin{array}{l}0.014 * \\
*\end{array}$ & \\
\hline & $(0.009)$ & & $(0.0356)$ & $(0.011)$ & & $(0.005)$ & \\
\hline \multirow[t]{2}{*}{ No. of active borrowers } & $0.01053 * *$ & & & $\begin{array}{l}0.012 * \\
*\end{array}$ & & $\begin{array}{l}0.021^{*} \\
*\end{array}$ & \\
\hline & $(0.0051)$ & & & $(0.006)$ & & $(0.008)$ & \\
\hline \multirow[t]{2}{*}{ Value of savings } & & $0.014 * *$ & $-0.099 * *$ & & $\begin{array}{l}0.011 * \\
*\end{array}$ & & $\begin{array}{l}0.014 * \\
* *\end{array}$ \\
\hline & & $(0.005371)$ & $(0.02081)$ & & $(0.005)$ & & $(0.005)$ \\
\hline \multirow{3}{*}{$\begin{array}{l}\text { No. of people with high school } \\
\text { education completion }\end{array}$} & 0.1010 & $0.125 * *$ & & & & & \\
\hline & $(0.0511)$ & $(0.05000)$ & & & & & \\
\hline & $0.0044^{*}$ & $0.0040^{*}$ & & & & & \\
\hline Households by other for lighting & $(0.0036)$ & $(0.0038)$ & & & & & \\
\hline \multirow[t]{2}{*}{ One room in household } & & & $-0.037 * *$ & & & & \\
\hline & & & $(0.0178)$ & & & & \\
\hline \multirow[t]{2}{*}{ Livestock ownership } & & & & $\begin{array}{l}0.684 * \\
* *\end{array}$ & $\begin{array}{l}0.649 * \\
* *\end{array}$ & $\begin{array}{l}0.794 * \\
* *\end{array}$ & $\begin{array}{l}0.812 * \\
* *\end{array}$ \\
\hline & & & & $(0.038)$ & $(0.045)$ & $(0.053)$ & $(0.051)$ \\
\hline Ownership of house & & & & $\begin{array}{l}0.035^{*} \\
* *\end{array}$ & $\begin{array}{l}0.040^{*} \\
* *\end{array}$ & $\begin{array}{l}0.028 * \\
* *\end{array}$ & $\begin{array}{l}0.033 * \\
* *\end{array}$ \\
\hline \multirow{3}{*}{ Ownership of household assets } & & & & $(0.010)$ & $(0.012)$ & $(0.014)$ & $(0.014)$ \\
\hline & & & & $\begin{array}{l}0.022 * \\
* *\end{array}$ & $\begin{array}{l}0.022 * \\
*\end{array}$ & $\begin{array}{l}0.028^{*} \\
* *\end{array}$ & $\begin{array}{l}0.025 * \\
* *\end{array}$ \\
\hline & & & & $(0.001)$ & $(0.018)$ & $(0.005)$ & $(0.012)$ \\
\hline Provincial dummy & Yes & Yes & Yes & Yes & Yes & Yes & Yes \\
\hline Time dummy & Yes & Yes & Yes & Yes & Yes & Yes & Yes \\
\hline \multirow[t]{2}{*}{ Constant } & & $3.038 * *$ & $5.273^{* *}$ & $\begin{array}{l}1.144 * \\
* *\end{array}$ & $\begin{array}{l}1.194 * \\
* *\end{array}$ & $\begin{array}{l}0.395^{*} \\
* *\end{array}$ & $\begin{array}{l}0.283^{*} \\
*\end{array}$ \\
\hline & & $(1.21771)$ & $(0.3451)$ & $(0.135)$ & $(0.157)$ & $(0.189)$ & $(0.179)$ \\
\hline No. of observations & 253 & 237 & 209 & 254 & 238 & 254 & 238 \\
\hline Adj. $R^{2}$ & 0.618 & 0.593 & 0.513 & 0.628 & 0.609 & 0.628 & 0.609 \\
\hline
\end{tabular}

Notes. Robust clustered standard errors are in parenthesis. All variables are in natural logarithm. Coefficients are obtained using the fixed effect panel approach. A time dummy and a district dummy are included in the estimation. ${ }^{* * *} \mathrm{p}<0.01 ; * * \mathrm{p}<0.05 ; * \mathrm{p}<0.10$. 


\section{Macrothink}

Table 10 demonstrates the impact of microfinance on a different measure of food consumption and livestock ownership. Food Consumption is a proxy for the proportion of households who spend money on food items, such as cereal, milk, eggs, meat, and fruit. The three microfinance measures have a positive impact on the consumption of food and the ownership of livestock, and the estimated coefficients are statistically significant at $1 \%$ and $5 \%$ levels. These results suggest that microfinance can be used as a vehicle for improving food consumption and ownership of household assets.

Table 10. Fixed effect panel regressions (health and living standard; income and food consumption) - Continued

\begin{tabular}{llll}
\hline \multicolumn{2}{l}{ Consumption of food } & \multicolumn{1}{l}{ Livestock ownership } \\
\hline Gross loan portfolio & $(1)$ & $(2)$ & $(3)$ \\
No. of active borrowers & $0.0610^{* *}$ & & $0.0567^{* *}$ \\
& $(0.0267)$ & & $0.0255)$ \\
Value of savings & $0.01053^{* * *}$ & & $(0.0178)$ \\
& $(0.0051)$ & & $0.099^{* * *}$ \\
Livestock ownership & & $0.014^{* * *}$ & $(0.02081)$ \\
& & $(0.005371)$ & \\
Ownership of house & $0.01043^{* *}$ & $0.022^{* *}$ & \\
& $(0.0049)$ & $(0.009)$ & $0.028^{* *}$ \\
Ownership of household assets & $0.1010^{* *}$ & $0.125^{* *}$ & $(0.014)$ \\
& $(0.0511)$ & $(0.05000)$ & \\
log(household income) & & & \\
Dummy Variables & & & \\
Provincial Dummy & $0.00449^{* * *}$ & $0.0040^{* * *}$ & \\
Time Dummy & $(0.0036)$ & $(0.00387)$ & Yes \\
Constant & & & Yes \\
& Yes & Yes & $5.273^{* * *}$ \\
\hline No. of observations & Yes & Yes & $(0.3451)$ \\
Adj. R & & $3.038^{* * *}$ & 209 \\
\hline
\end{tabular}

Notes. Robust clustered standard errors are in parenthesis. All variables are in natural logarithm. Coefficients are obtained using the fixed effect panel approach. A time dummy and a district dummy are included in the estimation. ${ }^{* * *} \mathrm{p}<0.01 ; * * \mathrm{p}<0.05 ;{ }^{*} \mathrm{p}<0.10$. 
Table 11. Fixed effect panel regressions (others)

\begin{tabular}{|c|c|c|c|c|c|c|}
\hline & \multicolumn{2}{|c|}{ Ownership of house } & \multicolumn{2}{|c|}{ Attendance of primary school } & \multicolumn{2}{|c|}{ Repayment of existing loans } \\
\hline & (1) & (2) & (3) & (4) & (5) & (6) \\
\hline \multirow[t]{2}{*}{ Gross loan portfolio } & $0.532 * * *$ & & $0.0639 * *$ & & $0.0665^{* *}$ & \\
\hline & $(0.155)$ & & $(0.0266)$ & & $(0.0265)$ & \\
\hline \multirow[t]{2}{*}{ No. of active borrowers } & $0.014 * *$ & & $0.022 * * *$ & & $0.039 * * *$ & \\
\hline & $(0.007)$ & & $(0.008)$ & & $(0.008)$ & \\
\hline \multirow[t]{2}{*}{ Value of savings } & & $0.016 * * *$ & & $0.018 * * *$ & & $0.019 * * *$ \\
\hline & & $(0.005)$ & & $(0.006)$ & & $(0.007)$ \\
\hline \multirow[t]{2}{*}{ Monthly wage } & $0.401 * * *$ & $0.355 * * *$ & $0.440 * * *$ & $0.417 * * *$ & $0.452 * * *$ & $0.449 * * *$ \\
\hline & $(0.051)$ & $(0.057)$ & $(0.058)$ & $(0.069)$ & $(0.059)$ & $(0.071)$ \\
\hline Provincial Dummy & Yes & Yes & Yes & Yes & Yes & Yes \\
\hline Time Dummy & Yes & Yes & Yes & Yes & Yes & Yes \\
\hline \multirow[t]{2}{*}{ Constant } & $2.680 * * *$ & $2.721 * * *$ & $2.372 * * *$ & $2.360 * * *$ & $1.78 * *$ & $1.827 * * *$ \\
\hline & $(0.179)$ & $(0.206)$ & $(0.207)$ & $(0.247)$ & $(0.211)$ & $(0.254)$ \\
\hline No. of observations & 254 & 238 & 254 & 238 & 254 & 238 \\
\hline Adj. $R^{2}$ & 0.642 & 0.640 & 0.688 & 0.669 & 0.715 & 0.672 \\
\hline
\end{tabular}

Notes. Robust clustered standard errors are in parenthesis. All variables are in natural logarithm. Coefficients are obtained using the fixed effect panel approach. A time dummy and a district dummy are included in the estimation. $* * * \mathrm{p}<0.01 ; * * \mathrm{p}<0.05 ; * \mathrm{p}<0.10$

Table 11 illustrates the impact of microfinance activities on house ownership, primary school attendance, and repayment of existing loans. The table demonstrates that, with the help of microfinance, people are more likely to buy and own houses, enroll children in schools, and repay existing loans. For example, the estimated coefficients show that a $10 \%$ increase in the number of active borrowers can raise house ownership by $0.14 \%$, raise attendance of primary education by $0.22 \%$, and increase repayment of existing loans by $0.39 \%$.

Finally, we examine whether the results remain consistent after using different methods for treating outliers. We rerun a regression with the 6th to 94th percentiles; this sample and observations differ, as we drop below the 5th and above the 95th percentile for poverty variables. We find that the results remain very similar to the previous ones.

\section{Conclusion and policy implications}

The objective of this paper is to examine the effect of microfinance programs on poverty alleviation by using multidimensional poverty measures in Pakistan. We find a statistically significant impact of microfinance on education, health, household income, food consumption, ownership of household assets, and so on. This result suggests that the development of microfinance is an efficient tool for both welfare improvement and poverty alleviation in Pakistan. These results appear robust with various specifications of empirical models. 
Although the results of this study show that there is a positive impact of microfinance on poverty alleviation, certain issues limit the active role of microfinance in poverty reduction. To improve microfinance schemes for better outcomes in Pakistan, it is recommended that a regulatory authority for the financial sector support microfinance banks to improve their working efficiency and outreach in rural areas and ensure that loans are disbursed to genuine clients based on realistic terms and conditions.

There are many other issues we need to consider. The size of loans should be increased to meet the borrowers' requirements. More opportunities for loans should be provided to poor people. Borrowers should be trained and educated properly for better loan utilization. Microfinance should establish entrepreneurship and conduct workshops on establishing and operating potential businesses. Microfinance should introduce education insurance for poor children so that they can continue their education even in extreme circumstances. Microfinance institutions should train their staff and recruit local staff who know the community well. Microfinance needs to expand into untapped areas, especially rural and slum areas. Moreover, microfinance should be considered as one of the major components of measuring the level of economic development of the country.

\section{References}

Ahmad, S., Naveed, M. S., \& Ghafoor, A. (2004). "Role of micro finance in alleviating rural poverty: A case study of Khushhali Bank Program in Rahim Yar Khan [Pakistan]". International Journal of Agriculture and Biology (Pakistan), 6(2), 426-428.

Ali, A. G., \& Thorbecke, E. (2000). The state and path of poverty in Sub-Saharan Africa: some preliminary results. Journal of African Economies, (Supplement 1), 9-40. https://doi.org/10.1093/jafeco/9.Supplement_1.9

Amjad, R., \& Kemal, A. R. (1997). Macroeconomic policies and their impact on poverty alleviation in Pakistan. The Pakistan Development Review, 39-68. https://doi.org/10.30541/v36ilpp.39-68

Barboza, G., \& Trejos, S. (2009). Micro credit in Chiapas, México: Poverty reduction through group lending. Journal of Business Ethics, 88(2), 283-299. https://doi.org/10.1007/s10551-009-0286-7

Brau, J. C., \& Woller, G. M. (2004). Microfinance: A comprehensive review of the existing literature. The Journal of Entrepreneurial Finance, 9(1), 1.

Coleman, B. E. (1999). The impact of group lending in Northeast Thailand. Journal of development economics, 60(1), 105-141. https://doi.org/10.1016/S0304-3878(99)00038-3

Collins, D., Morduch, J., Rutherford, S., \& Ruthven, O. (2009). Portfolios of the poor: how the world's poor live on $\$ 2$ a day. Princeton University Press.

Coleman, B. E. (2006). Microfinance in Northeast Thailand: Who Benefits and How Much. World Development, 34(9), 1612-1638. https://doi.org/10.1016/j.worlddev.2006.01.006

Conning, J., \& Udry, C. (2007). Rural financial markets in developing countries. Handbook 
of agricultural economics, 3, 2857-2908. https://doi.org/10.1016/S1574-0072(06)03056-8

Daley-Harris, S. (2002). Pathways out of poverty: Innovations in microfinance for the poorest families. Kumarian Press.

Doocy, S., Teferra, S., Norell, D., \& Burnham, G. (2005). Credit program outcomes: coping capacity and nutritional status in the food insecure context of Ethiopia. Social Science \& Medicine, 60(10), 2371-2382. https://doi.org/10.1016/j.socscimed.2004.10.025

Doyle, K. (1998). Microfinance in the wake of conflict: Challenges and opportunities. Microenterprise Best Practices.

Elbers, C., Lanjouw, J. O., \& Lanjouw, P. (2003). Micro-level estimation of poverty and inequality. Econometrica, 71(1), 355-364. https://doi.org/10.1111/1468-0262.00399

Gopalan, S. S. (2007). Micro-finance and its Contributions to Health Care Access (A Study of Self-Help Groups (SHGS) in Kerala). Health and Population Department of Kerala on Health and Population, 134, 149.

Hossain, M. (1988). Credit for alleviation of rural poverty: The Grameen Bank in Bangladesh (Vol. 65). Intl Food Policy Res Inst.

Johnson, S., \& Rogaly, B. (1997). Microfinance and poverty reduction. Oxfam. https://doi.org/10.3362/9780855988005

Khandker, S R. (2005). Microfinance and poverty: Evidence using panel data from Bangladesh. The World Bank Economic Review, 19(2), 263-286. https://doi.org/10.1093/wber/lhi008

Khandker, S. R., \& Pitt, M. M. (1998). The Impact of Group - Based Credit Programs on Poor Households in Bangladesh: Does the Gender of Participants Matter? Journal of political economy, 106(5), 958-996. https://doi.org/10.1086/250037

Manalo, M. S. (2003). Microfinance institutions' response in conflict environments: Eritrea-Savings and Micro Credit Program, West Bank and Gaza-Palestine for Credit and Development, Haiti-Micro Crédit National, SA. The World Bank.

Miller, J., \& Martinez, R. (2006). Championship league: An overview of 80 leading Latin American providers of microfinance. Microbanking Bulletin, (April).

Morduch, J. (1999). The microfinance promise. Journal of economic literature, 37(4), 1569-1614. https://doi.org/10.1257/jel.37.4.1569

Morduch, Jonathan (1999). The role of subsidies in microfinance: evidence from the Grameen Bank. Journal of development economics, 60(1), 229-248. https://doi.org/10.1016/S0304-3878(99)00042-5

Morduch, J. (2000). The microfinance schism. World development, 28(4), 617-629. https://doi.org/10.1016/S0305-750X(99)00151-5

Nagarajan, G., \& McNulty, M. (2004). Microfinance amid conflict: Taking stock of available 
literature. Accelerated Microenterprise Advancement Project. Washington, DC: USAID.

Osmani, L. N. K. (2007). .A Breakthrough in Women's Bargaining Power: the impact of microcredit. Journal of International Development, 19(5), 695-716. https://doi.org/10.1002/jid.1356

Pitt, M. M., Khandker, S. R., \& Cartwright, J. (2006). Empowering women with micro finance: Evidence from Bangladesh. Economic Development and Cultural Change, 54(4), 791-831. https://doi.org/10.1086/503580

Psacharopoulos, G., \& Woodhall, M. (1993). Education for development. Oxford University Press.

Rhyne, E. (1998). The yin and yang of microfinance: Reaching the poor and sustainability. Micro Banking Bulletin, 2(1), 6-8.

Swain, R. B., \& Floro, M. (2008). Effect of Microfinance on Vulnerability, Poverty and Risk in Low Income Households. Working Papers 2008-02, American University, Department of Economics.

Sharma, M., \& Zeller, M. (1999). Placement and outreach of group-based credit organizations: The cases of ASA, BRAC, and PROSHIKA in Bangladesh. World Development, 27(12), 2123-2136. https://doi.org/10.1016/S0305-750X(99)00106-0

Soulama, S. (2005). Micro-finance, poverty and development. Archives contemporaries.

Stephens, B., \& Tazi, H. (2006). Performance and Transparency: A Survey of Microfinance in South Asia. Micro banking Bulletin.

Wright, G. (2000). Microfinance systems: Designing quality financial services for the poor. London: Zed books.

\section{Notes}

Note 1. In Pakistan, microfinance institutions are divided almost equally into two institutional forms: microfinance banks (MFBs) with a more commercial orientation and non-governmental organizations (NGOs). A future study may attempt to compare commercialized MFBs with the traditional NGO model in terms of practice, outreach, mission, and the experience of clients.

Note 2. The PMN has a close association with CGAP, the Microfinance Information Exchange, and the Micro Banking Bulletin.

Note 3. The PSLM gathers data from the Household Integrated Economic Survey on food consumption, savings' level, employment status, and sources of income.

Note 4. To address this issue, we could fill the missing values through linear interpolation. However, this technique is not suitable for the PSLM data due to large variations in the data. The PSLM data contradict the assumption of linear interpolation, in which the data follow a predictable line. 


\section{Macrothink}

Journal of Asian Development

ISSN 2377-9594 2019, Vol. 5, No. 3

Note 5. Due to their meager incomes, most rural Pakistanis do not consult any formal physicians. In emergencies, they usually seek informal consultants, such as Hakeem or Jarrah.

Note 6. Because of meager incomes, most rural households cannot afford to eat three meals per day. Sometimes they eat only two meals per day and cannot afford fruit.

\section{Copyright Disclaimer}

Copyright for this article is retained by the author(s), with first publication rights granted to the journal.

This is an open-access article distributed under the terms and conditions of the Creative Commons Attribution license (http://creativecommons.org/licenses/by/4.0/). 